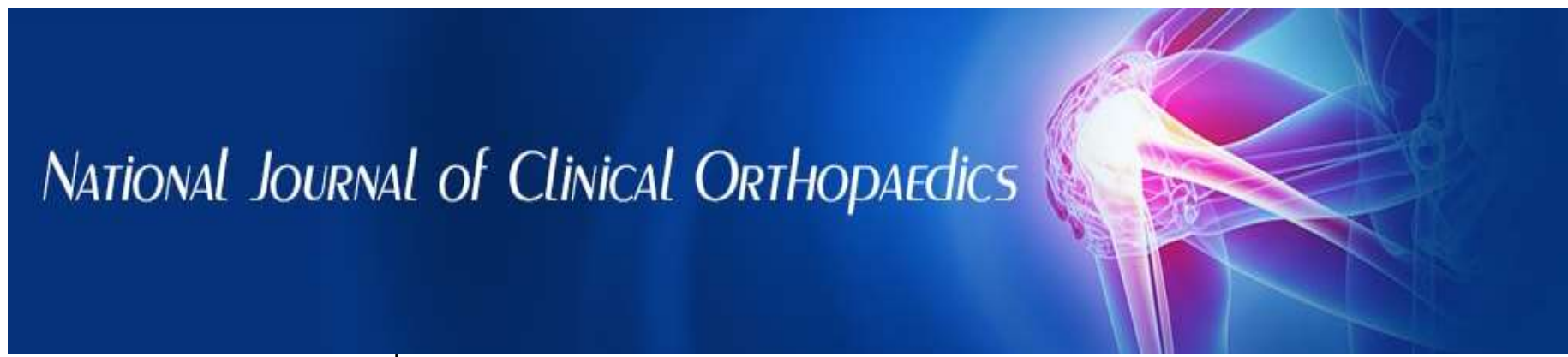

ISSN (P): 2521-3466

ISSN (E): 2521-3474

(C) Clinical Orthopaedics www.orthoresearchjournal.com 2021; 5(2): 24-26

Received: 24-12-2020

Accepted: 28-01-2021

Maria Dakou

Department of Physiotherapy,

Faculty of Health Sciences

International Hellenic University -

Alexander Campus P.O. Box 141,

57400 Sindos, Thessaloniki, Greece

\section{Paris Iakovidis}

Department of Physiotherapy, Faculty of Health Sciences

International Hellenic University -

Alexander Campus P.O. Box 141,

57400 Sindos, Thessaloniki, Greece

\section{Dimitrios Lytras}

Department of Physiotherapy,

Faculty of Health Sciences

International Hellenic University -

Alexander Campus P.O. Box 141,

57400 Sindos, Thessaloniki, Greece

\section{Ioannis Kottaras}

Department of Physiotherapy

Faculty of Health Sciences

International Hellenic University -

Alexander Campus P.O. Box 141,

57400 Sindos, Thessaloniki, Greece

\section{Anastasios Kottaras}

Department of Physiotherapy,

Faculty of Health Sciences

International Hellenic University -

Alexander Campus P.O. Box 141,

57400 Sindos, Thessaloniki, Greece

\section{Georgios Chasapis}

Department of Physiotherapy,

Faculty of Health Sciences

International Hellenic University -

Alexander Campus P.O. Box 141,

57400 Sindos, Thessaloniki, Greece

\section{The effect of physiotherapy in the treatment of piriformis syndrome: A narrative review}

\author{
Maria Dakou, Paris Iakovidis, Dimitrios Lytras, Ioannis Kottaras, \\ Anastasios Kottaras and Georgios Chasapis
}

DOI: https://doi.org/10.33545/orthor.2021.v5.i2a.278

\begin{abstract}
Piriformis syndrome (PS) is a common sciatic nerve entrapment syndrome caused by muscle spasm or other inflammatory processes in the piriformis muscle area, that lead to compression of the sciatic nerve resulting in the classic sciatica symptomatology. It manifests by pain in the dorsal surface of the thigh that reflects in the leg area, weakness and sensory disorders. The purpose of this review was to search the current literature on physiotherapy approaches to the treatment of symptoms of patients with PS. The PubMed, Science Direct, EBSCO and Google Scholar databases were searched with different combinations of the following keywords: Piriformis syndrome, physiotherapy, rehabilitation. The review included clinical studies, systematic reviews and case studies. From the results of this review, it appeared that there is not a large number of studies on this topic while in the existing studies that were included in this review, the number of participants was very small. The results of this review also show that the various manual techniques have a positive effect on the treatment of PS when combined with pharmacotherapy. In conclusion, the mobilization of the sciatic nerve as well as the strengthening of the muscles adjacent to the piriformis muscle, such as the adductor muscles, contribute significantly to the improvement of the symptoms of the patients, while best results are shown in combination with appropriate medication.
\end{abstract}

Keywords: Piriformis syndrome, physiotherapy, rehabilitation, resistance exercises

\section{Introduction}

Piriformis syndrome (PS) refers to the compression of the sciatic nerve at its exit point in the gluteal region after the sacroiliac ligament as it passes under the piriformis muscle ${ }^{[1]}$. It is caused by compression of the nerve due to muscle spasm or other inflammatory process of the muscle ${ }^{[1,2]}$. Patients with PS often experience pain and burning sensation in the back of the leg. In addition, these patients often experience numbness in the glutes, as well as a tingling sensation when moving the hip ${ }^{[3]}$. The main causes of PS are trauma to the hip area that can lead to piriformis muscle edema, piriformis muscle hypertrophy from excessive exercise (mainly seen in athletes during training or competition) and compression of the piriformis muscle by prolonged sitting ${ }^{[4,5]}$.

Epidemiologically, PS is responsible for $0.3-6 \%$ of all cases of low back pain and in most cases, patients present the typical sciatica symptoms. PS syndrome affects about 2.4 million of the 40 million low back pain and sciatica cases that occur annually ${ }^{[6]}$. Most often PS occurs mainly in middle-aged patients, while the ratio of women to men affected is $6: 1^{[7]}$.

The clinical evaluation for the diagnosis of this syndrome includes the conduct of specific stress tests for the piriformis muscle that aim to reproduce the symptoms during the physical examination ${ }^{[2]}$. At the same time, external pressure is applied manually by the therapist in order to fully and clearly assess the symptoms ${ }^{[8]}$.

The treatment of the syndrome initially includes rest of the patient and administration of appropriate medication (muscle relaxants, non-steroidal anti-inflammatory drugs, injections of corticosteroids peripheral to the piriformis muscle etc.) ${ }^{[7]}$, while the application of physiotherapy means offers significant relief from the symptoms ${ }^{[3]}$. The aim of this review was to search the current literature on the various physiotherapy approaches to the treatment and control of symptoms of PS.
Corresponding Author: Maria Dakou

Department of Physiotherapy, Faculty of Health Sciences International Hellenic University Alexander Campus P.O. Box 141, 57400 Sindos, Thessaloniki, Greece 


\section{Method}

The PubMed, ScienceDirect, EBSCO and Google Scholar databases were searched. The following keyword combinations and Boolean operators were used in the search: Piriformis syndrome AND physiotherapy AND rehabilitation. This review included clinical trials, systematic reviews and case studies involving the application of physiotherapy agents or techniques either individually or in combination with medication.

\section{Results}

This review included six articles. Below are their main findings.

\section{Literature review}

In the study of Fishman et al. ${ }^{[3]}$, the effect of the combination of physiotherapy and local injection with botulinum neurotoxin type B in the treatment of PS was investigated. The study involved 27 people with PS. The measurements included the serial H-reflex tests in flexion, adduction, and internal rotation, Visual Analog Scale (VAS) for pain and adverse effects at baseline and at the $2^{\text {nd }}, 4^{\text {th }}, 8^{\text {th }}$ and $12^{\text {th }}$ weeks. In this study, four intervention groups were formed, in which 5000, 75000, 10000 or 12500 units of botulinum toxin type B were injected. The physiotherapy program included ultrasound, hot and cold compresses, myofascial release and McKenzie exercises twice a week for a total of three months. The results of the study showed that the control of flexion and rotation of the hip was significantly increased. Overall, 24 of the 27 patients in the study experienced $50 \%$ pain relief compared to before this combination therapy. The researchers concluded that physiotherapy in combination with the injection of 12500 units of botulinum neurotoxin type $\mathrm{B}$ is safe and shows the best results in treating symptoms in patients with PS.

Other researchers have focused their research interest on therapeutic exercise, the association of the syndrome with the presence of muscle imbalances in the hip area and the selective strengthening of specific muscle groups. In the case study of Tonley et al., ${ }^{[9]}$ the effect of strengthening the adductor and external rotator muscles of the hip in a patient with PS was assessed. Following the intervention, the patient reported elimination of pain, while the score of the initial Lower Extremity Functional Scale questionnaire score improved from $65 / 80$ to $80 / 80$. Furthermore, based on the performance of a step-down task, peak hip internal rotation decreased from $12.8^{\circ}$ to $5.9^{\circ}$ and peak hip adduction from $15.9^{\circ}$ to $5.8^{\circ}$. The results of this study showed that targeted therapeutic exercise can eliminate the symptoms of the syndrome, when it is a secondary manifestation of a muscle imbalance and address the real cause that led to the disorder.

Michel et al. ${ }^{[1]}$ studied the efficacy of a diagnostic tool for the syndrome in the evaluation of 250 patients with PS, while also proposing an appropriate strategy for its treatment. The treatment protocol involved a combination of muscle relaxants, medication and physiotherapy techniques for three sessions per week. These techniques involved stretching the muscles, similar to the evaluation procedure during the physical examination of patients, with specific stretching of the muscles surrounding the hip. Despite the program having an initial scheduled duration of six weeks, the patients were evaluated three months after the start of the intervention. The results shows that the combination of medication and rehabilitation treatments had a cure rate of $51.2 \%$, while $48.8 \%$ of patients did not respond to treatment and received a neurotoxin (Onabotulinum toxin type A).

Boyajian-O'Neill et al. ${ }^{[7]}$ in their review point out that there are many studies which include various physiotherapy approaches, in order to treat PS. The application of hot and cold compresses, therapeutic ultrasound, massage, exercises and the application of stretching, according to the authors, are interventions with very good results. More specifically, they note that the main goal of physiotherapy interventions is to alleviate the symptoms of patients while increasing the range of motion of the muscles and tendons, in order to increase support, muscle strength and consequently, the functioning of the individual. They also report that the training of the adductor muscles has had beneficial results in these patients. Finally, other approaches are the combined treatment regimens that include the application of physiotherapy with local injections.

Moreover, Probst et al. ${ }^{[2]}$ state in their review that the initial management of symptoms immediately after diagnosis is critical. The researchers point out that the basic guidance of the treatment is realized by measuring the length of the muscle and the strain of the nerve, with a characteristic clinical point being the increased tone of muscle relaxation. Thus, they suggest that therapists should focus on the central stabilization of the core, the strengthening of the hip, the correction of the pelvis and spine postures, which could predispose to the appearance of gluteal pain. This review highlights that sciatic nerve gliding can greatly help reduce lower limb pain and can also be used to monitor the condition of the patient during treatment.

Lastly, Nazlikul et al. ${ }^{[10]}$ studied the effect of physiotherapy approaches related to hip neural function, in order to control the functioning of patients with PS. The 102 participants were divided into two groups. The first received the neural treatment and the second was the control group, while all the patients followed specific exercises for stretching the piriformis muscle. Patients were evaluated with the VAS and the Oswestry Disability Index before and after treatment. The results of this study showed positive results in both groups, with a statistically greater improvement in the group that received the neural activation.

\section{Discussion-results}

There are many gaps in the knowledge about the pathophysiology, symptoms and treatment of PS, so as noted by most of the researchers included in this review, further research is needed on this condition. Additionally, it is also suggested for future studies to include larger samples. Existing studies involve too small a number of participants to safely draw conclusions. New imaging methods available for patients with PS can be used for the differential diagnosis of the condition ${ }^{[1,9]}$. Regarding treatment methods, symptomatic therapies provide the most benefits; predominantly pharmacotherapy ${ }^{[3]}$. As far as physiotherapy approach is concerned, the activation and strengthening of the muscles seems to show a positive correlation with the improvement of pain [9], as well as the gliding exercises for the sciatic nerve and the exercises for stretching the muscles and tendons adjacent to the hip [7] However, we have not identified a large number of studies that focus on the physiotherapy intervention in the treatment of PS. The issue requires further investigation.

\section{References}

1. Michel F, Decavel P, Toussirot E, et al. Syndrome du muscle piriforme: Critères diagnostiques et traitement à propos d'une série monocentrique de 250 patients. Ann Phys Rehabil Med. 2013;56(5):371-383. doi:10.1016/j.rehab.2013.04.003

2. Probst D, Stout A, Hunt D. Piriformis Syndrome: A Narrative Review of the Anatomy, Diagnosis, and 
Treatment. PM R. 2019;11:54-63. doi:10.1002/pmrj.12189

3. Fishman LM, Konnoth C, Rozner B. Botulinum Neurotoxin Type B and Physical Therapy in the Treatment of Piriformis Syndrome: A Dose-Finding Study. Am J Phys Med Rehabil 2004;83(1):42-52+60. doi:10.1097/01.PHM.0000104669.86076.30

4. Smoll NR. Variations of the piriformis and sciatic nerve with clinical consequence: A review. Clin Anat. 2010;23(1):8-17. doi:10.1002/ca.20893

5. Cassidy L, Walters A, Bubb K, Shoja MM, Tubbs RS, Loukas M. Piriformis syndrome: Implications of anatomical variations, diagnostic techniques, and treatment options. Surg Radiol Anat. 2012;34(6):479-486. doi:10.1007/s00276-012-0940-0

6. Norbury JW, Morris J, Warren KM, et al. Piriformis syndrome is a neuromuscular condition that remains poorly understood and often misdiagnosed. Diagnosis and Management of Piriformis Syndrome 2012, 24-27. http://practicalneurology.com/pdfs/PN0612_ExpertOpinion. pdf.

7. Boyajian-O’Neill LA, McClain RL, Coleman MK, Thomas PP. Diagnosis and management of piriformis syndrome: An osteopathic approach. J Am Osteopath Assoc 2008;108(11):657-664. doi:10.7556/jaoa.2008.108.11.657

8. Vassalou EE, Katonis P, Karantanas AH. Piriformis muscle syndrome: A cross-sectional imaging study in 116 patients and evaluation of therapeutic outcome. Eur Radiol 2018;28(2):447-458. doi:10.1007/s00330-017-4982-x

9. Tonley JC, Yun SM, Kochevar RJ, Dye JA, Farrokhi S, Powers CM. Treatment of an individual with piriformis syndrome focusing on hip muscle strengthening and movement reeducation: A case report. J Orthop Sports Phys Ther 2010;40(2):103-111. doi:10.2519/jospt.2010.3108

10. Nazlikul H, Ural FG, Oztürk GT, Oztürk ADT. Evaluation of neural therapy effect in patients with piriformis syndrome. J Back Musculoskelet Rehabil 2018;31(6):11051110. doi:10.3233/BMR-170980 